\title{
The rapid emergence of high level gentamicin resistance in enterococci
}

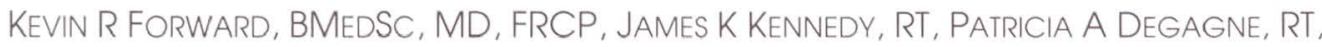 \\ KATHY R BARTLET, BSC, GODFREY KM HARDING, BSC, MD, FRCP
}

\begin{abstract}
The proportion of enterococci isolated from blood and urine cultures that were highly resistant to gentamicin and streptomycin were determined. No blood or urine isolates highly resistant to gentamicin were seen in 1983, whereas by 1986-87 25\% of blood and $17 \%$ of urine isolates were highly resistant. The
\end{abstract}

rapid emergence of gentamicin resistance has serious implications for patients with life threatening enterococcal disease. Can J Infect Dis 1990;1(3):97-100

Key Words: Enterococci, Enterococcus faecalis, Gentamicin. High level resistance, Streptomycin
E NTEROCOCCI REPRESENT AN INCREASINGLY IMPORtant cause of infection, especially in hospitalized patients. In recent years, there has been an increase in both the absolute number of infections and the proportion of nosocomial infections due to Enterococcus species (1-3). Enterococcal bacteremia is the cause of significant morbidity and mortality, especially when associated with endo-

Departments of Laboratory Medicine and Medicine, St Boniface General Hospital; and Departments of Medical Microbiology and Medicine, University of Manitoba, Winnipeg. Manitoba

Correspondence and reprints: Dr KR Forward. Department of Microbiology. Victoria General Hospital, 5788 University Avenue, Halifax, Nova Scotia B3H 1 V8

Received for publication January 26, 1990. Accepted July 27. 1990 carditis or meningitis (2,4-7). Life threatening enterococcal infections are usually treated with a combination of an aminoglycoside and either a penicillin or vancomycin (2). These combinations have also been used to prevent enterococcal endocarditis in predisposed patients following genitourinary manipulation.

In recent years, strains highly resistant to aminoglycosides have been reported with increasing frequency (8-13). These strains are not susceptible to the synergistic action of an aminoglycoside and either a penicillin or vancomycin (13). In order to determine whether such strains were prevalent in Manitoba, a study of blood and urine isolates recovered at two large Winnipeg teaching hospitals over a five year period was conducted. 
TABLE 1

Summary of enterococcal strains tested

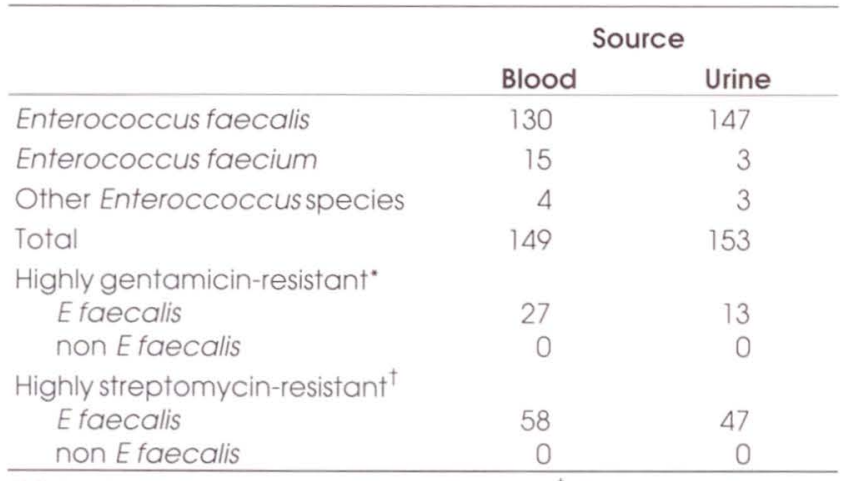

"Minimal inhibitory concentration $>500 \mathrm{mg} / \mathrm{L} ;{ }^{\dagger}$ Minimal inhibitory concentration $>2000 \mathrm{mg} / \mathrm{L}$

\section{MATERIALS AND METHODS}

Enterococcus species isolated from patients cared for at St Boniface General Hospital and the Health Sciences Centre over a six year period (1982-87) were studied. Blood and urinary isolates had been collected and stocked in skim milk at $-70^{\circ} \mathrm{C}$. Urinary isolates were predominantly from patients seen in outpatient infectious diseases clinics. Duplicate isolates from the same patient were excluded from analysis. Isolates were speciated using the identification scheme of Falklam (14). Susceptibilities to gentamicin and streptomycin were determined using the agar dilution method on Mueller-Hinton agar (Scott Laboratories Inc, Carson, California). Strains were considered to possess high level resistance to gentamicin if the minimal inhibitory concentration (MIC) was in excess of $500 \mathrm{mg} / \mathrm{L}$ and to streptomycin if the MIC was in excess of $2000 \mathrm{mg} / \mathrm{L}$. In order to validate results of susceptibility testing, time-kill synergy studies were performed on strains isolated from blood with MICs within two dilutions of the breakpoint. Time-kill synergy studies were performed in cation-supplemented
Mueller-Hinton broth using gentamicin $5 \mathrm{mg} / \mathrm{L}$ or streptomycin $15 \mathrm{mg} / \mathrm{L}$, each with penicillin 10 $\mathrm{mg} / \mathrm{L}$. Synergy was said to be present when there was a 100-fold reduction in colony count in the presence of both agents, compared with each individually.

\section{RESULTS}

Susceptibilities of 149 blood culture isolates and 153 urinary isolates of Enterococcus species were determined. Ninety-three per cent of blood and $98 \%$ of urine isolates were revived from stock cultures. Results are shown in Table 1 and Figure 1. One hundred and thirty blood culture isolates were $E$ faecalis, 15 were $E$ faecium and four were other species. Of the 153 urinary isolates, 147 were $E$ faecalis, three were $E$ faecium and three were other species. In the first two years of the study, there were no blood culture isolates highly resistant to gentamicin. In 1986 and 1987, 17 of 69 isolates $(24.6 \%)$ recovered from blood were resistant to gentamicin. During the same period of time, nine of 54 strains (16.7\%) from urine were highly resistant to gentamicin. Three of 16 blood culture isolates $(18.7 \%)$ were highly resistant to streptomycin in 1983, whereas 10 of 24 (41.6\%) were highly resistant by 1987 . The proportion of isolates from urine highly resistant to streptomycin did not change significantly over the period studied, and ranged from $16.7 \%$ in 1983 to $40.7 \%$ in 1987 $\left(\chi^{2} 3.55 ; P=0.62\right)$.

Of the 27 gentamicin-resistant $E$ faecalis strains recovered from blood, 24 were highly resistant to streptomycin; three were not. There were 13 urinary isolates highly resistant to gentamicin, eight of which were also highly streptomycinresistant. In each case, when penicillin-gentamicin synergy testing was performed, results confirmed that the MICs used correctly identified highly resistant strains.
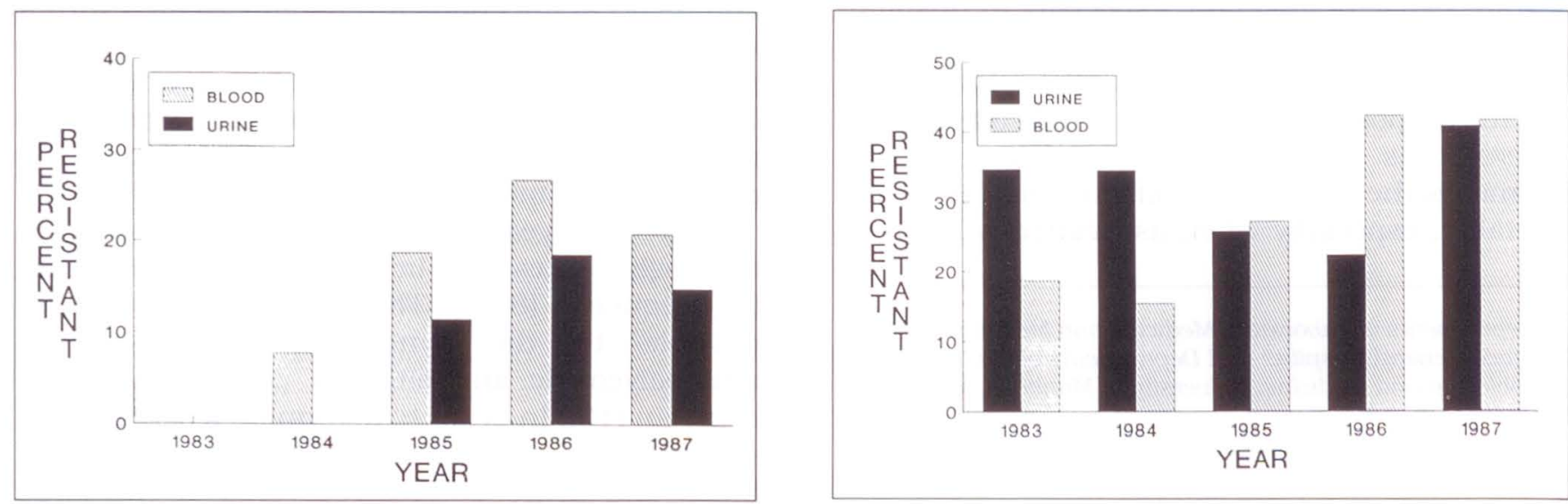

Figure 1) Percentage of Enterococcus species strains isolated from blood or urine and highly resistant to gentamicin (left) (minimal inhibitory concentration $>500 \mathrm{mg} / \mathrm{L}$ ) or streptomycin (right) (minimal inhibitory concentration $>2000 \mathrm{mg} / \mathrm{L}$ ) 


\section{DISCUSSION}

The use of both an aminoglycoside and a penicillin together has been considered standard therapy for the treatment of enterococcal infections since synergy between both classes of antibiotic was first described (15). Strains highly resistant to streptomycin have been recognized for a number of years. However, strains highly resistant to gentamicin were not recognized prior to 1979 (8). Since that time, enterococci highly resistant to gentamicin have been recognized with increasing frequency. These strains produce aminoglycoside-inactivating enzymes capable of adenylation, phosphorylation or acetylation of aminoglycosides and render them resistant to the synergistic interaction of aminoglycoside and cell wall-active agents such as the penicillins and vancomycin (16).

Patients with serious infections due to highly resistant Enterococcus species may not respond to combined therapy and may be unnecessarily exposed to potentially toxic aminoglycosides. Therefore, such isolates should be identified by the clinical laboratory as highly resistant.

The usual breakpoints for determining susceptibility of other Gram-positive and Gram-negative bacteria are inappropriate for determining the presence of aminoglycoside high level resistance (17). Other methods have been developed to detect this form of resistance. The first is the use of a disk diffusion test with high potency antibiotic-impregnated disks $(18,19)$. These disks are not yet commercially available and must be produced inhouse. A second method involves determining the ability of enterococci to grow in a broth containing either $500 \mathrm{mg} / \mathrm{L}$ of gentamicin or $2000 \mathrm{mg} / \mathrm{L}$ of streptomycin (17). This method has been incorporated into at least one commercially available system (American Microscan Inc, Sacramento, California).

The prevalence of highly resistant enterococci in Canadian hospitals has not been well established. Mederski-Samoraj et al (20) found that

\section{REFERENCES}

1. Jones RN. Gram positive superinfections following beta-lactam chemotherapy: Significance of the enterococcus. Infection 1985;13(Suppl 1):S81-8.

2. Maki DG, Agger WA. Enterococcal bacteremia: Clinical features, the risk of endocarditis and management. Medicine 1988;67:248-66.

3. Morrison AJ, Wenzel RP. Nosocomial urinary tract infections due to enterococcus: Ten years experience at a university hospital. Arch Intern Med 1986; 146:1549-51.

4. Malone DA, Wagner RA, Myers JP, et al. Enterococcal bacteremia in two large community teaching hospitals. Am J Med 1986;81:601-6.
$15.5 \%$ of blood culture isolates and $12.5 \%$ of isolates from other sterile body sites were highly resistant to gentamicin. They noted that prevalence varied considerably between hospitals. Clinicians should be made aware of the presence of strains highly resistant to aminoglycosides, especially those isolated from blood and cerebrospinal fluid. It is important to note that three blood culture isolates highly resistant to gentamicin were susceptible to streptomycin and that streptomycin can probably be used in combination with a penicillin when severely ill patients are encountered. Although alternative treatment regimens have not yet been established, other therapies may be considered in such cases. No two agents have shown synergy against strains of $E$ faecalis highly resistant to aminoglycosides. Studies in the authors' laboratory have shown that the new lipopeptide antibiotic daptomycin tested at $5 \mathrm{mg} / \mathrm{L}$ is more rapidly bactericidal than penicillin, vancomycin or teicoplanin in vitro (unpublished observations). Whether this observation will equate to in vivo efficacy in humans remains to be determined. Clinicians caring for seriously ill patients with infection due to highly resistant enterococci may choose to administer longer courses of antibiotics, may be more likely to intervene surgically and may provide more careful follow-up.

In summary, highly resistant $E$ faecalis were recovered with increasing frequency from both the urinary tract of a predominantly outpatient population and from the blood of hospitalized patients. Clinicians should be aware of this emerging problem and laboratories should provide appropriate susceptibility data on enterococci isolated from blood or cerebrospinal fluid, or repeatedly from other extra-urinary sites in order to optimize the care of infected patients. Treatment of urinary tract infections due to enterococci usually does not require an aminoglycoside, and screening for high level resistance is probably unnecessary in this setting.

5. Bayer AS, Seidel JS, Yoshikawa TT. Group D enterococcal meningitis: Clinical and therapeutic considerations with report of three cases and review of the literature. Arch Intern Med 1976; 136:883-6.

6. Mandell GL, Kaye D, Levison ME, et al. Enterococcal endocarditis: An analysis of 38 patients observed at the New York Hospital-Cornel Medical Centre. Arch Intern Med 1970;125:258-64.

7. Barrall DT, Kenney PR, Slotman GJ, et al. Enterococcal bacteremia in surgical patients. Arch Surg 1985; 120:57-63.

8. Horodniceanu TM, Bougueleret L, El-Sohl N, et al. High level, plasmid-borne resistance to gentamicin 
in Streptococcus faecalis subsp. zymogenes. Antimicrob Agents Chemother 1979;16:686-9.

9. Zervos MJ, Dembinski S, Mikesell T, et al. High level resistance to gentamicin in Streptococcus faecalis: Risk factors and evidence for exogenous acquisition of infection. $J$ Infect Dis 1986; 153:1075-83.

10. Zervos MJ, Kaufmann CA, Terasse PM, et al. Epidemiology of nosocomial infection caused by gentamicin-resistant Streptococcus faecalis. Ann Intern Med 1987;106:687-91.

11. Ikeda DP, Barry AL, Anderson SG. Emergence of Streptococcus faecalis isolates with high-level resistance to multiple aminocyclitol aminoglycosides. Diagn Microbiol Infect Dis 1984;2:171-7.

12. Zervos MJ, Terpenning MS, Schaberg DR, et al. High-level aminoglycoside-resistant enterococci: Colonization of nursing home and acute care hospital patients. Arch Intern Med 1987:147:1591-4.

13. Mederski-Samoraj BD, Murray BE. High-level resistance to gentamicin in clinical isolates of enterococci. J Infect Dis 1983; 147:751-7.

14. Falklam RR, Carey RB. Streptococci and Aerococci. In: Lennette EH, ed. Manual of Clinical
Microbiology. Washington: American Society for Microbiology, 1985:154-75.

15. Sandiford HD, deMaine JB, Kirby WM. Antibiotic synergy of enterococci. Arch Intern Med 1970;126:255-9.

16. Davies JE. Aminoglycoside-aminocyclitol antibiotics and their modifying enzymes. In: Lorian $\mathrm{V}$, ed. Antibiotics in Laboratory Medicine, 2nd edn. Baltimore: Williams and Wilkins, 1986:790-804

17. Zervos MJ, Patterson JE, Edberg S, et al. Singleconcentration broth microdilution test for detection of high-level aminoglycoside resistance in enterococci. J Clin Microbiol 1987;25:2443-4.

18. Rosenthal SL, Freundlich LF. An aminoglycoside disk sensitivity test for use with enterococci. J Antimicrob Chemother 1982;10:459-62.

19. Sahm DF, Torres C. High-content aminoglycoside disks for determining aminoglycoside-penicillin synergy against Enterococcus faecalis. J Clin Microbiol 1988;26:257-60.

20. Mederski-Samoraj B, Simon A, Fuller S, et al. High-level aminoglycoside resistance of enterococcal isolates in Canada. 88th Annual Meeting Medical Society for Microbiology, Miami Beach, Florida, 1988;C307. (Abst) 


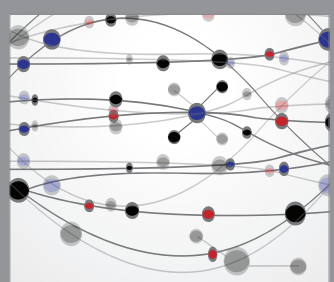

The Scientific World Journal
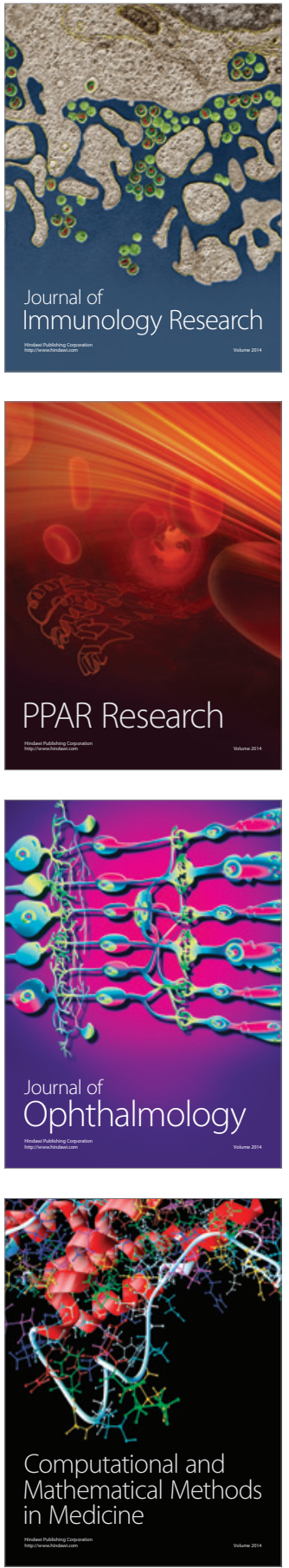

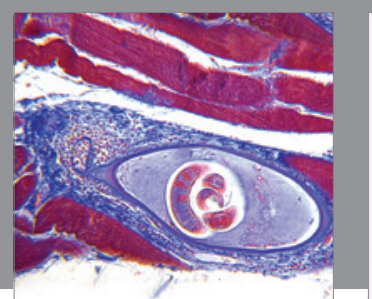

Gastroenterology Research and Practice

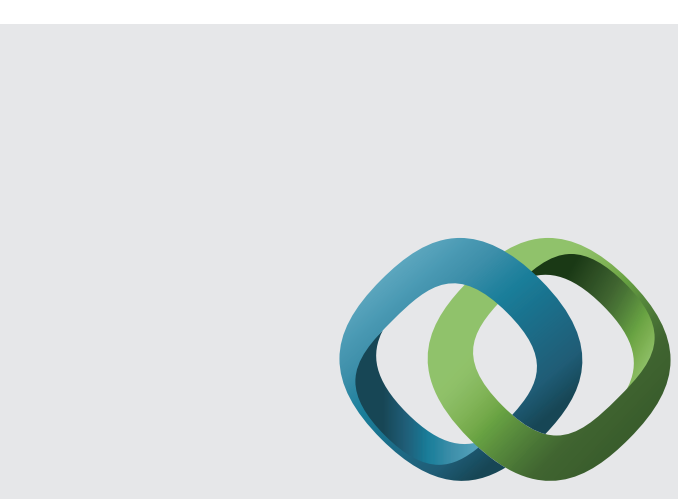

\section{Hindawi}

Submit your manuscripts at

http://www.hindawi.com
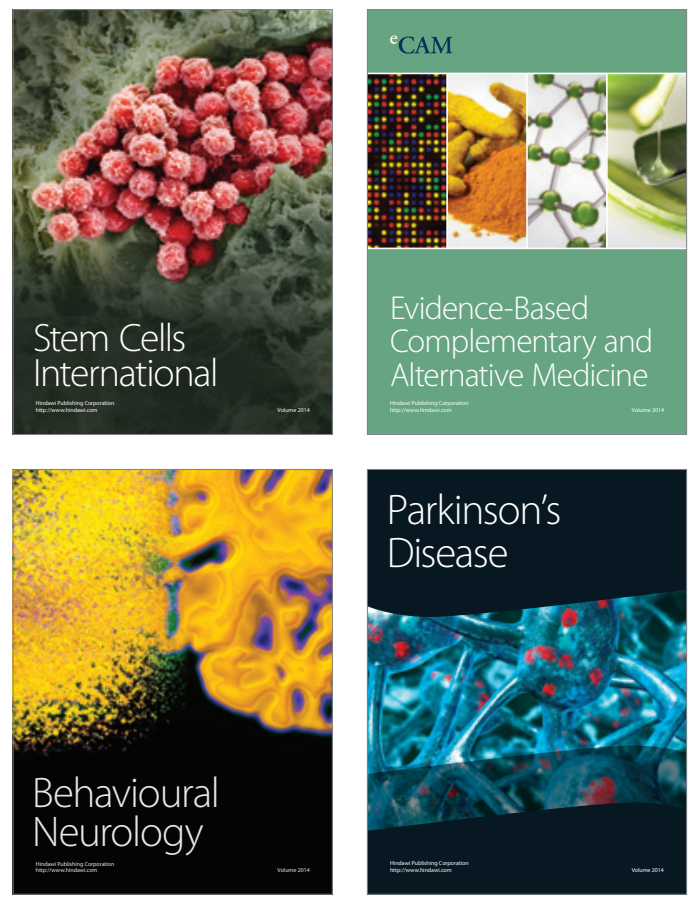
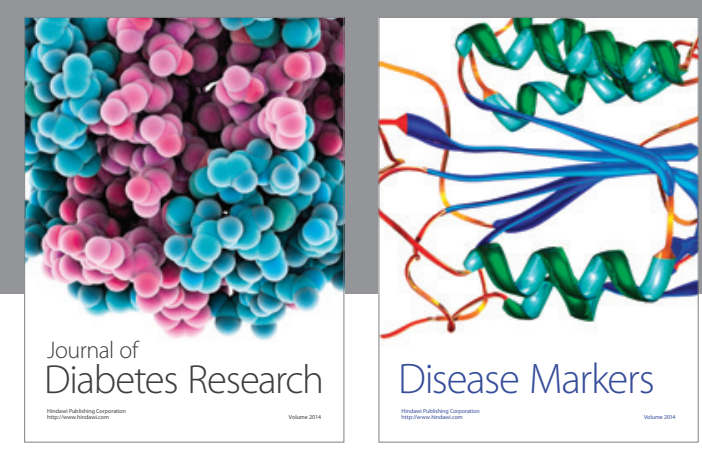

Disease Markers
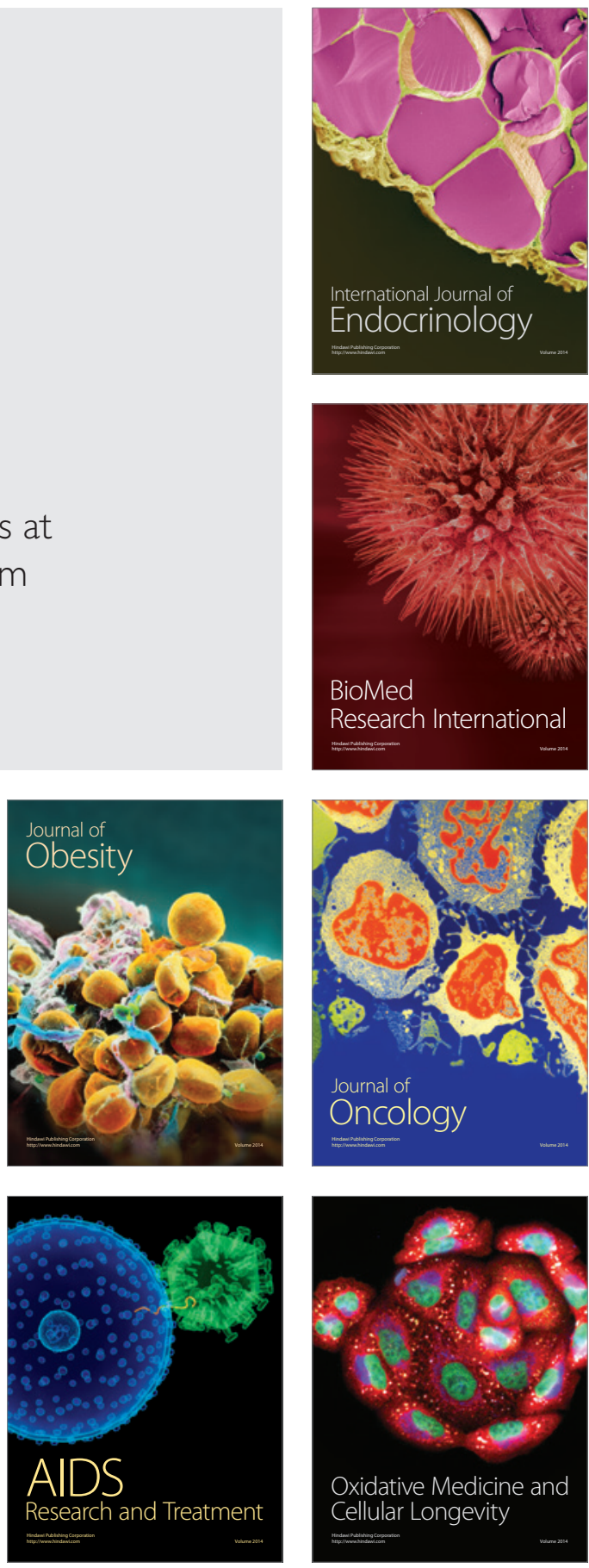